Louisiana State University

LSU Digital Commons

$1-1-2006$

\title{
Relations between Chinese mothers' parenting practices and social withdrawal in early childhood
}

\author{
Larry J. Nelson \\ Brigham Young University \\ Craig H. Hart \\ Brigham Young University \\ Bo Wu \\ Brigham Young University \\ Chongming Yang \\ Brigham Young University \\ Susanne Olsen Roper \\ Brigham Young University
}

See next page for additional authors

Follow this and additional works at: https://digitalcommons.Isu.edu/biosci_pubs

\section{Recommended Citation}

Nelson, L., Hart, C., Wu, B., Yang, C., Roper, S., \& Jin, S. (2006). Relations between Chinese mothers' parenting practices and social withdrawal in early childhood. International Journal of Behavioral Development, 30 (3), 261-271. https://doi.org/10.1177/0165025406066761 


\section{Authors}

Larry J. Nelson, Craig H. Hart, Bo Wu, Chongming Yang, Susanne Olsen Roper, and Shenghua Jin 


\title{
Relations between Chinese mothers' parenting practices and social withdrawal in early childhood
}

\author{
Larry J. Nelson, Craig H. Hart, Bo Wu, \\ Chongming Yang, and Susanne Olsen Roper \\ Brigham Young University, USA
}

\author{
Shenghua Jin \\ Beijing Normal University, China
}

\begin{abstract}
Researchers have identified specific parenting practices used by parents of preschoolers in mainland China (e.g., physical coercion, overprotection, shaming, directiveness, encouragement of modesty). Some of the intrusive practices have been linked to social withdrawal in western societies (e.g., United States, Canada). It seemed important to examine these associations in China because recent research suggests that young Chinese children who exhibit wariness in peer settings may be at risk for negative outcomes such as peer rejection. Therefore, the purpose of this study was to examine the relation between Chinese parenting practices and preschoolers' social withdrawal. Mothers of preschool-age children from mainland China $(N=446)$ completed self-report parenting questionnaires. Teachers rated children's reticent, solitary-passive, solitary-active, and modest behaviors. Results showed that (a) maternal directiveness was positively associated with reticent behavior in girls and negatively associated with solitary-passive behavior in boys, (b) maternal overprotection, for girls, was positively related to both reticent behavior and solitary-passive behavior, and negatively related to modest behavior, (c) coercion was positively associated with solitary-active and reticent behavior in girls, and (d) shaming was positively related to all forms of withdrawn behaviors in boys and girls, as well as positively related to modest behavior in boys.
\end{abstract}

Keywords: Chinese parenting; intrusive parenting; modest behaviour; parenting practices; social withdrawal; withdrawn subtypes

Recent interest in better understanding Chinese and western parenting has resulted in numerous studies designed to illuminate etic commonalities and emic cultural nuances in parenting cognitions, styles, and practices (e.g., Cheah \& Rubin, 2004; Chen, Wu, Chen, Wang, \& Cen, 2001; Nelson, Nelson, Hart, Yang \& Jin, in press; Wu et al., 2002). Researchers have also recently started to examine whether the same relations between parenting and child outcomes in western cultures exist in Eastern cultures such as China. These attempts to find patterns which either vary across cultures or may be universal to all human beings have led to mixed findings and disagreement.

For example, recent studies have yielded similarly measureable parenting constructs in mainland China and North America (e.g., Chen, Liu, Li, Cen, et al., 2000; Porter et al., 2005; Wu et al., 2002). Research has also revealed meaningful cross-cultural similarities in the outcomes associated with Chinese parenting styles, including links between authoritative parenting and positive child outcomes (e.g., peer preference, social competence, school achievement), and relations between both authoritarian and permissive parenting, respectively, and negative child outcomes (e.g., social maladjustment, peer rejection, aggression; Chang, Lansford, Schwartz, \&
Farver, 2004; Chen, Dong \& Zou, 1997; Chen, Liu, \& Li, 2000; Yang et al., 2004). These similarities in the relations between parenting and child outcomes in western and Eastern cultures have led some researchers to conclude that parenting styles may serve similar functions in child rearing in mainland China as they do in western cultures (Chen et al., 1997).

However, other researchers disagree and point out an equal number of dissimilarities. For example, in European American samples in the United States, academic performance tends to be facilitated by authoritative parenting (e.g., Steinberg, Lamborn, Darling, Mounts, \& Dornbusch, 1994) while research with Chinese immigrant families suggests that authoritarian parenting fosters academic performance. Specifically, Chao (1994) found that Chinese parents were more authoritarian than European American parents (and conversely European American parents were more authoritative) and had children who performed better academically. Similar results have been found in studies examining parenting and academic achievements of adolescents in Hong Kong, the United States, and Australia (Leung, Lau, \& Lam, 1998) and Chinese Americans (Chao, 2001).

These inconsistent findings have led to disagreement concerning whether constructs developed in one culture (i.e.,
Correspondence should be sent to Larry J. Nelson, PhD, Marriage, Family, \& Human Development, 2091 JFSB, Brigham Young University, Provo, UT 84602, USA; e-mail: larry_nelson@byu.edu

We are grateful to Dr Dahua Wang at Beijing Normal University for helping coordinate part of the Chinese data collection. Portions of this article were presented at the biennial meetings of the Society for
Research in Child Development, Atlanta, Georgia, April 2005. Funding for this study was provided by several sources at Brigham Young University: the College of Family, Home, and Social Sciences, the Camilla Eyring Kimball Endowment, the Family Studies Center, and the Zina Young Williams Card Professorship awarded to Craig H. Hart. 
North America) can be applied to other cultures (i.e., China). For example, Chao (1994) argues that the conceptualization of authoritarian parenting ignores the purpose of parental control and does not capture the main idea of Asian authoritarian parenting. Immigrant Chinese parents put more emphasis on educational achievement and set high standards for their children, and, therefore, their controlling behavior actually pushes their children to reach educational success (Chao, 2001; Chao \& Sue, 1996). However, when measured specifically as harsh, coercive parental control, authoritarian parenting has been associated with more aggressive behavior with peers and lower academic achievement in mainland China (e.g., Chen, Dong, \& Zhou, 1997; Nelson, Hart, Yang, Olsen, $\&$ Jin, 2006). As Chao and Tseng (2002) have recently noted, “. . . the effects of control on child well-being for Asians appear to depend on the way control is defined such that indigenous notions have positive effects and a domineering control primarily has negative effects among groups of Asian and European descent" (p. 77).

Concerns arising from similar and discrepant findings depending on how constructs are culturally defined and measured reflect the emic-etic problem (e.g., Berry, 1989), which evaluates whether behaviors under investigation are emic (arising from the culture) or etic (similar across cultures). Studying Chinese parenting by applying Baumrind's (1967, 1971) typologies would be an etic approach, but Chao (1994) argued that using an emic approach would lead to a better understanding of parenting and child outcomes in China. Indeed, researchers have started to focus less on broad parenting styles and more on specific parenting practices in different cultures. A parenting practice, a component of parenting style, includes parental behaviors defined by specific context and socialization goals (Darling \& Steinberg, 1993), such as helping children develop social and academic skills, or conform to social expectations (Hart, Newell, \& Olsen, 2003).

Recent research has examined specific parenting practices that are reflective of Chinese values and beliefs. For example, in a study of mothers of preschoolers, Wu et al. (2002) examined five parenting constructs stemming from the Chinese socialization literature which were highly valued in China: encouragement of modest behavior (e.g., discouraging a child from showing off), parental protection (e.g., expecting a child to play close by), shaming/love withdrawal (e.g., telling a child they should be ashamed when misbehaving), directiveness (e.g., demanding a child do things), and maternal involvement (e.g., taking care of child needs). Wu and colleagues found that these five parenting practices were also empirically identifiable in a North American sample but relatively independent from the constructs emphasized in America (e.g., warmth/acceptance, democratic participation). Results showed that Chinese mothers scored higher than American mothers on parenting practices emphasized by Chinese parents except for maternal involvement. They also scored higher on the use of physical coercion, lower on warmth/acceptance and democratic participation, and about equal on the use of reasoningoriented regulation (cf. Chen et al., 2001; Porter et al., 2005). These findings were consistent with results of work conducted with parents of toddlers. Specifically, Chen and colleagues (1998) found that, compared to mothers in Canada, Chinese mothers were less accepting, more rejecting, more likely to encourage children to achieve, more protective of their children, and more punishment oriented. In another study examining cultural differences in specific parenting practices,
Lin and $\mathrm{Fu}$ (1990) found that Chinese parents scored higher on parental control and emphasized achievement more than Caucasian-American parents.

As noted by many observers of Chinese parenting (e.g., Ho, 1986; Tseng \& Wu, 1985), shaming is a prevalent Chinese socialization practice designed to help children be sensitive to the perceptions of others and to teach them to avoid future behaviors that would bring shame or embarrassment to the family. Fung (1999), for example, recently described how shaming is practiced by Tiawanese parents in the socialization of their children's moral behavior. This construct, as explicated by Fung, overlaps somewhat with Western notions of psychological control (e.g., Barber, 2002; Hart et al., 1998) and includes elements of guilt induction, love withdrawal, guilt laden warnings of punishment, along with explicit statements about being embarrassed and ashamed of child misbehavior. Although shaming is generally thought to threaten children's self-esteem in western society, a discretionary use of shame in Confucian philosophy is thought to help children regulate and enact their behavior in culturally appropriate modest, tactful, restrained, respectful, and sensitive ways (Fung, 1999).

Based on these findings which show that Chinese parents do use specific practices (e.g., physical coercion, overprotection, shaming, directiveness), it seems important to examine the child behaviors that are associated with them. Some of these intrusive practices (i.e., coercion, overprotectiveness and directiveness) have been linked to social withdrawal in Western society (e.g., see Burgess, Rubin, Cheah, \& Nelson, 2001). Recent research in China suggests that young children who exhibit wariness in peer settings may be at risk for negative outcomes such as peer rejection (Hart et al., 2000). Yet, it is unknown whether childhood withdrawal is associated in any way with Chinese parenting. Hence, the purpose of this study was to examine the relation between Chinese parenting practices and preschooler's social withdrawal.

\section{Parenting and social withdrawal}

Researchers have found several important links between parenting and fearful, inhibited, and withdrawn behaviors in children in western cultures. Most notably, emerging evidence suggests that oversolicitous parenting (i.e., overly protective/ controlling, intrusive behavior) is linked to children's social withdrawal (see Burgess et al., 2001, for a complete review). For example, Rubin, Burgess, and Hastings (2002) found that mothers of inhibited toddlers (age 2) who engaged in intrusive parenting behaviors (e.g., unsolicited intervention) had children who engaged in high amounts of reticent/wary behavior at age 4 . There was no relation between age-2 inhibition and age- 4 reticence for children whose mothers were not intrusive. Similarly, Rubin, Nelson, Hastings, and Asendorpf (1999) found that parental perceptions of child shyness/social wariness at age 2 predicted both mothers' and fathers' expressed lack of encouragement of independence. Taken together, these studies suggest that some parents may respond to perceived wariness in their children with parental behaviors (i.e., intrusive, overprotective parenting) that actually exacerbate the problem by not allowing children opportunities to develop regulatory and coping skills to deal with their social anxieties.

Researchers have not yet directly examined the association between parenting practices and withdrawal in early childhood 
in mainland China. However, Cheah and Rubin (2004) examined the parenting beliefs of mothers of preschool age children in both the United States and mainland China. They found that mothers in both countries regarded socially withdrawn behaviors in their children negatively. In addition, while mothers in both countries reported they would react anxiously to hypothetical vignettes depicting their children engaged in withdrawn behaviors, Chinese mothers were less likely to report that they would respond in a way that focused on the needs of their child. Specifically, given collectivistic cultural orientations, Chinese mothers were more concerned with how their child fit into the peer group than how the child was feeling.

Given this concern with successfully being a part of the group in order to maintain social order and interpersonal harmony (Chen et al., 1998), Chinese mothers may be fairly direct in their parenting practices. Indeed, given that Chinese parents already tend to favor protective, directive, and shaming parenting practices (e.g., Fung, 1999; Wu et al., 2002), it would seem important to examine the relation between greater usage of these practices and social withdrawal in young children. The specific parenting practices of interest in this study were encouragement of modest behavior, overprotection, coercion, directiveness, and shaming. While past studies in North America have examined the linkages between these types of parenting practices to global conceptualizations of social withdrawal, more recent studies have focused on multifaceted aspects of this construct or subtypes of withdrawal (e.g., Rubin et al., 2002), which may well apply to Chinese preschoolers' peer group behavior (Hart et al., 2000).

\section{Social withdrawal}

Based on research conducted in Western societies, it appears that social interaction, especially interaction with peers, is extremely important for children's social development (see Rubin, Bukowski, \& Parker, 1998, for a review). Based on this, concern exists for those children who do not interact with their peers. Specifically, researchers have demonstrated that social withdrawal is related to many negative outcomes for children such as low self-esteem, depression, anxiety, and loneliness (see Rubin, Burgess, \& Coplan, 2002, for a review). In the Chinese view, social withdrawal may also be considered problematic because it does not reflect the group-oriented values of a collectivistic culture.

According to Rubin et al. (2002), social withdrawal refers to "the consistent (across situations and over time) display of all forms of solitary behavior when encountering familiar/ unfamiliar peers” (p. 330). As captured in this definition, multiple forms of withdrawn behaviors have been identified. According to Asendorpf (1990), the different types of withdrawal are a result of different motivational tendencies to either approach or avoid social interaction. Coplan, Prakash, O'Neil, and Armer (2004) tested this notion and empirically distinguished between conflicted shyness (i.e., a desire to interact socially coupled with social fear and anxiety) and social disinterest (i.e., lack of a strong motivation to engage in social interaction). It is believed that these two types of social motivation are manifested in different behaviors. Specifically, three subtypes of social withdrawal are observable during early childhood: reticence, solitary-passive withdrawal, and solitaryactive behavior (e.g., Coplan, et al., 1994; Coplan \& Rubin, 1998; Rubin \& Mills, 1988; Rubin, Hymel \& Mills, 1989).
Solitary-passive withdrawal is characterized by the quiet exploration of objects and constructive activities while playing alone (e.g., building with blocks, reading books; Coplan et al., 1994). While once thought to reflect a low social approach tendency and a low social avoidance motivation (Asendorpf, 1990), recent research calls into question the exact underlying meaning of solitary-passive behavior (e.g., Coplan et al., 2004; Spinrad et al., 2004). However, in early childhood in Western cultures, the behavior has been found to be positively associated with competent problem solving with peers, task persistence, performance on object-oriented tasks, and emotion regulation (Coplan et al., 1994; Coplan \& Rubin, 1998; Rubin, 1982; Rubin, Coplan, Fox, \& Calkins, 1995).

Solitary-active behavior is characterized by solitary-functional play (repeated sensorimotor actions with or without objects such as hopping or skipping) or by solitary dramatic/pretend play (Coplan et al., 1994; Rubin, 1982). When displayed in early childhood, this behavior tends to reflect social incompetence and immaturity and leads to isolation by the peer group (Rubin, 1982, Rubin et al., 1995). For example, solitary-active behavior has been found to be negatively associated with positive group interactions, peer conversations, perspective-taking ability, and problem-solving ability, as well as positively associated with impulsivity, social maladjustment, emotion dysregulation, maladaptive social information-processing patterns, and peer rejection (Coplan et al., 1994; Rubin, 1982; Rubin et al., 1995).

Reticence is defined as frequent onlooking and unoccupied behaviors in both familiar and unfamiliar social contexts (Coplan et al., 1994). This type of social withdrawal reflects an approach-avoidance conflict in that reticent children want to approach others, but they have a simultaneous avoidance tendency. During early childhood, this subtype of withdrawal in Western cultures has been found to be associated with anxious-fearful and hovering behaviors, peer rejection, negative emotion regulation, and internalizing disorders (e.g., Coplan \& Rubin, 1998; Hart, DeWolf, \& Burts, 1993; Hart et al., 2000; Rubin et al., 1995).

\section{Social withdrawal and gender differences}

There is evidence to suggest that withdrawn behavior may be related to more adjustment problems in boys than girls in western cultures. Specifically, researchers have not found significant gender differences in the display (i.e., frequency) of various nonsocial behaviors (Coplan, Molina, Lagace-Seguin, \& Wichmann, 2001; Coplan et al., 1994; Coplan \& Rubin, 1998; Rubin, 1982), but they have found gender differences in the outcomes associated with the display of these behaviors. For example, in preschoolers, shy boys tend to have more behavior problems than shy girls (Simpson \& Stevenson-Hinde, 1985). In kindergarten, solitary-passive behavior in boys has been negatively associated with social competence and positively associated with internalizing disorders (Coplan et al., 2001). Taken together, it appears that nonsocial behavior in boys may have different adjustment implications than the same behavior displayed by girls, but little if any research has been conducted to examine possible gender differences in the relations between preschoolers' withdrawn subtypes and parenting practices in China.

Indeed, there is little extant research in general on the correlates of social withdrawal in China. Earlier work in the 1990s with older children and adolescents (Chen, Rubin, \& Li, 
1995; Chen, Rubin, \& Sun, 1992) suggests that shyness/sensitivity may be linked to positive outcomes (e.g., social competence, leadership, peer acceptance) because these behaviors reflect group-dependent, sensitive, and sociallyrestrained behaviors that may be more valued in the Chinese culture (see Chen et al., 2000; Ho, 1986; Lau, 1996). However, in a more recent 2002 cohort, shyness was linked to peer rejection, school problems and depression in Chinese elementary-school age children, suggesting the possibility of societal transition effects over the past decade (Chen, Cen, Li, \& He, 2005). Schwartz, Chang, and Farver (2001) also recently found a link between submissive-withdrawal and peer victimization in 11-year-old Chinese children. Furthermore, the limited work that has been done examining withdrawal in early childhood in an earlier 1995 mainland Chinese sample suggests that social withdrawal, in particular reticence, is associated with peer rejection (Hart et al., 2000).

The discrepant findings across the past decade may also be due, in part, to different operational definitions of withdrawal. For example, Chen and colleagues include behaviors such as "easily hurt feelings" and "usually sad" to identify shyness. These items seem to indicate oversensitivity or internal anxious social reactivity rather than social solitude and wariness (Chen et al., 2005). Others may confuse the culturally-valued notion of modesty (i.e., quiet, reserved, not boastful; Fung, 1999) as being withdrawn. In the current study, we attempt to address this issue by distinguishing between various types of withdrawal and modest behavior reflecting restrained, respectful, quiet, sensitive behavior.

\section{Hypotheses}

Based on research conducted in Western cultures that parental overprotection and intrusiveness appear to be linked to social withdrawal, it was hypothesized that similar associations would exist in China. While this study is unable to determine causal directions (i.e., parenting causes child behavior or vice versa), research in Western cultures suggests that the association between intrusive parenting and withdrawal holds regardless of whether child behavior elicits intrusive parenting (e.g., Rubin et al., 1999) or intrusive parenting leads to reticent behavior (e.g., Rubin et al., 2001). Therefore, it was expected that parenting practices that limit children's opportunities to practice social skills and regulate their own emotions would be positively associated with socially fearful and incompetent (i.e., immature and impulsive) behaviors. Specifically, it was anticipated that maternal overprotection, directiveness, and coercion would be positively associated with reticent and solitary-active behaviors but not solitary-passive behavior because it tends not to reflect either fear or immaturity (e.g., Coplan et al., 1994). There is very little research on withdrawal and gender in China upon which to make predictions based on gender. However, the research in Western cultures would suggest that there is greater concern for withdrawn behaviors in boys. Hence, it is expected that the relation between maternal overprotection, directiveness, and coercion and reticence and solitary-active behavior would be particularly strong for boys.

Next, the expectation exists for Chinese children to become sensitive to shame and to become aware of the opinions, judgments, and evaluations of others (Schoenhals, 1993). Hence, shaming is viewed as an important training technique for achieving socialization goals. As a result, Chinese parents may use it to both elicit desired behaviors from their children and correct behaviors deemed inappropriate. Specifically, shaming may be used in response to behaviors that are not conducive to group harmony (e.g., reticent and solitary-active behavior). Therefore, it was hypothesized that shaming would be associated with less of both reticence and solitary-active withdrawal for both boys and girls.

Finally, because we believed modesty (quiet, not showing off, not boasting), unlike withdrawal, reflects behavior that is conducive to being a part of a group, we did not expect associations between parental intrusive behaviors (directiveness, overprotection, coercion) and child modesty. However, we did expect positive correlations between child modesty and both parental encouragement of modesty and shaming because parents are likely to teach children to be modestly restrained in their behavior.

\section{Methods}

\section{Participants}

Participants in this study included 446 mothers of 219 boys and 227 girls from four full-day preschools in Beijing and one full-day preschool in Hangzhou of mainland China. Class sizes ranged from 20 to $26(M=22.3)$. At least $70 \%$ of children in each class were involved. The age of children ranged from 4 to 6 years (48-81 months), with an average of 5.2 years $(S D=$ .7) for boys and $5.3(S D=.8)$ for girls. Mothers ranged in age from 26 to 47 years $(M=33.2$ years; $S D=4$ years). Mothers were relatively well educated in that the average years of education was $13.2(S D=2.7)$. Head teachers of these children also participated in this study.

\section{Procedure}

Schools in China act "in loco parentis," and thus we were not allowed to obtain written parental permission (cf. Chen et al., 1992; Hart et al., 2000). However, school administrators helped arrange group meetings with the parents so that procedures could be explained. At these group meetings, teachers and parents were assured of confidentiality. The questionnaires were given to the parents and teachers along with instructions on how to complete them. They were allowed to take the questionnaires home and were asked to return them within one month.

\section{Measures}

Teacher and parent questionnaires were forward- and backtranslated by Chinese and American linguists who were fluent in both English and Chinese. Any discrepancies were discussed and necessary changes in wording were made.

Assessment of parental practices. Based on previous work (e.g., Wu et al., 2002), 18 items were selected and/or modified and created to measure the following constructs: encouragement of modesty (four items, e.g., "discourages child from showing off his/her skills and knowledge to get attention"); overprotection (four items; e.g., "tend to be overly involved in our child's activities"); shaming/psychological control (seven items also reflecting Fung, 1999, shaming conceptualizations; e.g., 
"make child feel guilty when our child doesn't meet our expectations"); coercion (three items; e.g., "spank when our child is disobedient"); and directiveness (four items; e.g., "tells our child how he/she should behave"). See Table 1 for a complete list of items included in each dimension. Instrument items that mothers completed were accompanied by a 5-point scale anchored by 1 (never) to 5 (always).

Teacher ratings. Teachers completed a battery of measures that tapped into a variety of child behaviors. When rating each child, teachers were instructed to "think about the child's present behavior relative to others in this age group that you know or have known." Instrument items were accompanied by a 3-point scale anchored by never (0) and very often (2). Previous work has demonstrated the ability of teachers to discriminate between the subtypes of withdrawal in China (Hart et al., 2000). The items reflecting each of the three withdrawn subtypes used in this previous work (e.g., Hart et al., 2000) were selected for use in this study (14 items total) plus an additional 4 items assessing modesty. Specifically, the withdrawn scales measured reticence (e.g., stares at other children without interacting; wanders aimlessly during free play; is fearful in approaching other children); solitary-passive (e.g., constructive activities such as blocks and puzzles away from peers); and solitary-active behavior (e.g., animate toys alone by pretending an inanimate object, such as a doll or stick, is alive). The modesty scale was made up of four items including "brags or boasts" and "flaunts his/her points of view strongly" (all modesty items were reverse coded so higher scores reflected greater modesty). See Table 3 for a complete list of items included in each dimension.

\section{Results}

The analyses for this study were carried out with the Mplus program in three steps. First, the measurement of parenting constructs was tested with a two-group (boys and girls) Confirmatory Factor Analysis (CFA) to establish measurement invariance across groups. Second, the measurement of child outcome constructs was tested with a two-group CFA to determine whether teacher-rated items were invariant across boys and girls. Third, the association of each parenting construct with child outcomes was estimated with a two-group structural equation modeling approach that included each parenting construct as the exogenous latent variable and each child outcome as the endogenous variable. In addition, given the rather large range of ages in the participants (4-6 years), child age was incorporated as a covariate to control for the variance in the subtypes of withdrawal associated with child age.

Because the data were collected with Likert scales and nonnormally distributed, all the observed variables were treated as ordinal (Lubke \& Muthén, 2004; Wright, 1999). The Mplus program has the ability to treat skewed Likert scales as ordinal categorical variables using polychoric correlations, instead of Pearson product-moment correlations, for structural equation modeling (Muthén \& Muthén, 2001). Some statisticians have argued that categorical analysis of such data is more

Table 1

Factor structure of parenting constructs

\begin{tabular}{|c|c|c|c|}
\hline \multirow[b]{2}{*}{ Parenting constructs } & \multirow[b]{2}{*}{ Measurement items } & \multicolumn{2}{|c|}{ Factor loadings } \\
\hline & & Girls & Boys \\
\hline \multicolumn{4}{|l|}{ Directiveness } \\
\hline & Tells our child how he/she should behave & .47 & .54 \\
\hline & Demands child do things & .59 & .69 \\
\hline & Wants to control whatever our child does & .81 & .71 \\
\hline & Tries to change our child & .61 & .54 \\
\hline \multicolumn{4}{|l|}{ Overprotection } \\
\hline & Tends to be overly involved in our child's activities & .75 & .60 \\
\hline & Tends to be overprotective with our child & .56 & .44 \\
\hline & It is important to supervise all of our child's activities & .41 & .51 \\
\hline & Readily intervenes if there is a chance that our child will fail at something & .69 & .58 \\
\hline \multicolumn{4}{|c|}{ 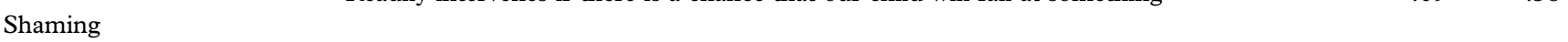 } \\
\hline & Tells child that he/she should be ashamed when misbehaving & .48 & .42 \\
\hline & Tells child we get embarrassed when he/she doesn't meet our expectations & .52 & .55 \\
\hline & Makes child feel guilty when our child doesn't meet our expectations & .51 & .48 \\
\hline & Brings up our child's past mistakes when criticizing him/her & .50 & .65 \\
\hline & Informs our child that punishment will find him/her when misbehavior occurs & .74 & .57 \\
\hline & Tells our child he/she is not as good as other children & .67 & .59 \\
\hline & Less friendly with our child if our child does not see things his/my way & .64 & .65 \\
\hline \multicolumn{4}{|c|}{ 20 } \\
\hline & Spanks when our child is disobedient & .83 & .78 \\
\hline & Grabs our child when being disobedient & .56 & .65 \\
\hline & Slaps our child when the child misbehaves & .82 & .83 \\
\hline \multicolumn{4}{|c|}{ Encouragement of modesty } \\
\hline & Discourages child from expressing his/her points of view around others & .70 & .61 \\
\hline & Discourages child from proudly acknowledging compliments or praise from friends or adults & .56 & .67 \\
\hline & Discourages child from appearing overconfident to others about his/her abilities & .58 & .60 \\
\hline & Discourages child from showing off his/her skills or knowledge to get attention & .66 & .79 \\
\hline
\end{tabular}


Table 2

Latent intercorrelations of parenting constructs

\begin{tabular}{|c|c|c|c|c|c|}
\hline Girls Boys & Directiveness & Overprotection & Shaming & Coercion & Encouragement of modesty \\
\hline Directiveness & & $.58^{\star}$ & $.75^{\star}$ & $.30^{\star}$ & -.03 \\
\hline Overprotection & $.49^{\star}$ & & $.41^{\star}$ & .05 & .07 \\
\hline Shaming & $.61^{\star}$ & $.43^{\star}$ & & $.47^{\star}$ & $-.26^{\star}$ \\
\hline Coercion & $.27^{\star}$ & .13 & $.50^{\star}$ & & -.00 \\
\hline Encouragement of modesty & $.22^{\star}$ & .18 & $-.26^{\star}$ & .08 & \\
\hline
\end{tabular}

Note. ${ }^{\star} p<.05$.

Table 3

Factor structure of child outcome constructs

\begin{tabular}{|c|c|c|c|}
\hline \multirow[b]{2}{*}{ Child outcome constructs } & \multirow[b]{2}{*}{ Measurement items } & \multicolumn{2}{|c|}{ Factor loadings } \\
\hline & & Girls & Boys \\
\hline \multicolumn{4}{|l|}{ Solitary-passive } \\
\hline & Would rather play alone & .87 & .93 \\
\hline & Does artwork by self, away from others & .88 & .82 \\
\hline & Does constructive activities alone (e.g., blocks, puzzles) & .88 & .88 \\
\hline & Reads books alone, away from others & .87 & .73 \\
\hline & Plays with toys by self rather than with other children & .93 & .91 \\
\hline \multicolumn{4}{|l|}{ Solitary-active } \\
\hline & Does pretend/dramatic play by self & .97 & .93 \\
\hline & Animates toys by self (e.g., pretends an inanimate object (doll or stick) is alive) & .85 & .82 \\
\hline & Does pretend/dramatic play with peers, but does not interact with them while doing so & .92 & .89 \\
\hline \multicolumn{4}{|c|}{ ק } \\
\hline & Wanders aimlessly during free play & .77 & .71 \\
\hline & Is off task and preoccupied & .76 & .76 \\
\hline & Appears to be doing nothing & .89 & .90 \\
\hline & Stares at other children without interacting with them & .91 & .96 \\
\hline & Is fearful in approaching other children & .75 & .77 \\
\hline & Watches other children play without joining in & .93 & .86 \\
\hline \multicolumn{4}{|l|}{ Modest reserved $\star$} \\
\hline & Flaunts his/her points of view strongly & .88 & .89 \\
\hline & Proudly acknowledges compliments or praise & .80 & .82 \\
\hline & Brags or boasts & .72 & .84 \\
\hline & Appears overconfident with his/her abilities & .81 & .89 \\
\hline
\end{tabular}

Note. ${ }^{\star}$ Items reverse coded.

appropriate than continuous analysis, in that it lends better estimates of pathways in SEM models (e.g., Coenders, Satorra, \& Saris, 1997). In particular, this assertion is based on the argument that treating ordinal data as if it were interval may produce distorted findings, especially when data is skewed. Indeed, comparative studies show that latent correlations are significantly attenuated and model fit is reduced when product-moment correlations of categorical variables, rather than polychoric correlations, are employed (see Yang et al., 2004, for a thorough review of relevant research). Accordingly, the measurement and structural model testing in this study employed ordinal analysis of the data.

First, a two-group (boys and girls) CFA was conducted to examine the measurement model of parenting constructs (i.e., directiveness, overprotection, shaming, coercion, encouragement of modesty). The following commonly cited Goodnessof-fit Indices were selected to indicate the model's fit: (a) Bentler's Comparative Fit Index (CFI), and (b) Tucker Lewis Indices (TLI), also known as Non Normed Fit Indices (NNFI). Models with CFI values greater than .90 have traditionally been considered acceptable models (Kline, 1998). The measurement model was found to fit the data very well with $\chi^{2}=226.75, \mathrm{df}=142, \mathrm{CFI}=.94, \mathrm{TLI}=.95$, RMSEA $=.05$. The standardized factor loadings of parenting constructs are listed in Table $1 .{ }^{1}$ As seen in Table 2, the constructs were relatively uncorrelated or only modestly correlated with the exception of a higher correlation between shaming and directiveness.

Measurement invariance of the factor loadings was tested by comparing a model with factor loadings constrained to be equal across the two groups (boys and girls) to a model without

\footnotetext{
${ }^{1}$ In Structural Equation Modeling (SEM), only three or four indicators are needed in order for the measurement model to be over-identified (Kline, 1998). Factor loadings in this study were acceptable based on prior studies indicating that they are typically underestimated in SEM when using Likert-type scaling. This is due to the treatment of the ordinal data (Bollen, 1989; Coenders \& Saris, 1997; Rigdon \& Ferguson, 1991). Factor loadings of .40 and above are deemed reliable when sample sizes are greater than 150 (Stevens, 1996). Coefficient alpha is a questionable indicator of reliability and is not typically reported in confirmatory factor analysis (Schmitt, 1996; Komaroff, 1997).
} 
such equality constraints. The chi-square difference corresponding to the difference in the degree of freedom showed that all the factor loading are equivalent with $\chi_{\text {dif }}^{2}=10.91, \mathrm{df}_{\mathrm{dif}}=14$, $p>.05$. Measurement invariance implied that the items used to measure the constructs performed equivalently for both boys and girls.

In the same fashion, the measurement model of child variables (solitary-passive, reticent, solitary-active, and modest behaviors) was also found to fit the data very well $\left(\chi^{2}=126.22\right.$, $d f=69, \mathrm{CFI}=.99, \mathrm{TLI}=.99, \mathrm{RMSEA}=.06)$. The standardized factor loadings are listed in Table 3. As in prior research using latent correlations rather than zero-order correlations (Hart et al., 2000), the constructs were highly related, with solitary-passive and solitary-active $(N=.90)$, solitary-passive and reticence $(N=.92)$, and solitary-active and reticence $(N$ $=.89)$ for girls and solitary-passive and solitary-active $(N=$ $.92)$, solitary-passive and reticence $(N=.91)$, and solitaryactive and reticence $(N=.90)$ for boys. Immodesty was only significantly correlated with reticence $(N=-.26)$ for girls and $(N=-.21)$ for boys. Measurement invariance was tested and found that all the items had invariant factor loadings $\left(\chi^{2}\right.$ dif $=$ 13.65, $\left.\mathrm{df}_{\mathrm{dif}}=8, p>.05\right)$.

Due to the high intercorrelations of the child outcome constructs, several alternative models in which two withdrawn constructs were treated as one while retaining all their indicators were sequentially compared with the model that had three withdrawal constructs. The chi-square difference tests showed that the model with three separate withdrawal factors fit the data much better than any other two-factor model.
Merging any two of the constructs worsened the fit significantly, with solitary-active behavior and solitary-passive behavior as one construct $\chi_{\text {dif }}^{2}=37.20, \mathrm{df}_{\text {dif }}=7, p<.00$, reticence and solitary-passive behavior as one construct $\chi^{2}{ }_{\text {dif }}=$ $24.64, \mathrm{df}_{\text {dif }}=5, p<.00$, and solitary-active and reticence as one construct $\chi_{\text {dif }}^{2}=51.51$, for $\mathrm{df}_{\mathrm{dif}}=5, p<.01$. Thus, the three constructs were well-distinguished, in spite of their high latent intercorrelations.

In the final structural equation model of the associations between parenting and child outcomes, the factor loadings of both parenting and child outcome constructs were constrained to be equal across the two groups, so as to reduce the number of parameters estimated and to increase the power of the parameters estimated. In addition, the associations of each parenting construct with child outcomes were tested to see if they were equivalent across both groups. This was done using chi-square differences to compare each structural equation model with another having equality constraints on the paths from the parenting construct to the child outcome construct. The final path estimates reported in Table 4 were based on models with equality constraints that had been tested across the two groups.

Child age was found to be negatively associated with reticence $(\gamma=-.18$ to $-.20, z>.1 .96, p<.05)$ and positively associated with modesty $(\gamma=.19$ to $.20, z>.1 .96, p<.05$; their magnitudes varied slightly with each respective parenting constructs across all the models). These findings suggested that older children displayed fewer reticent behaviors and more modest behaviors. However, the parenting constructs in this

Table 4

Standardized path coefficients of parenting constructs and child outcome constructs in each SEM model

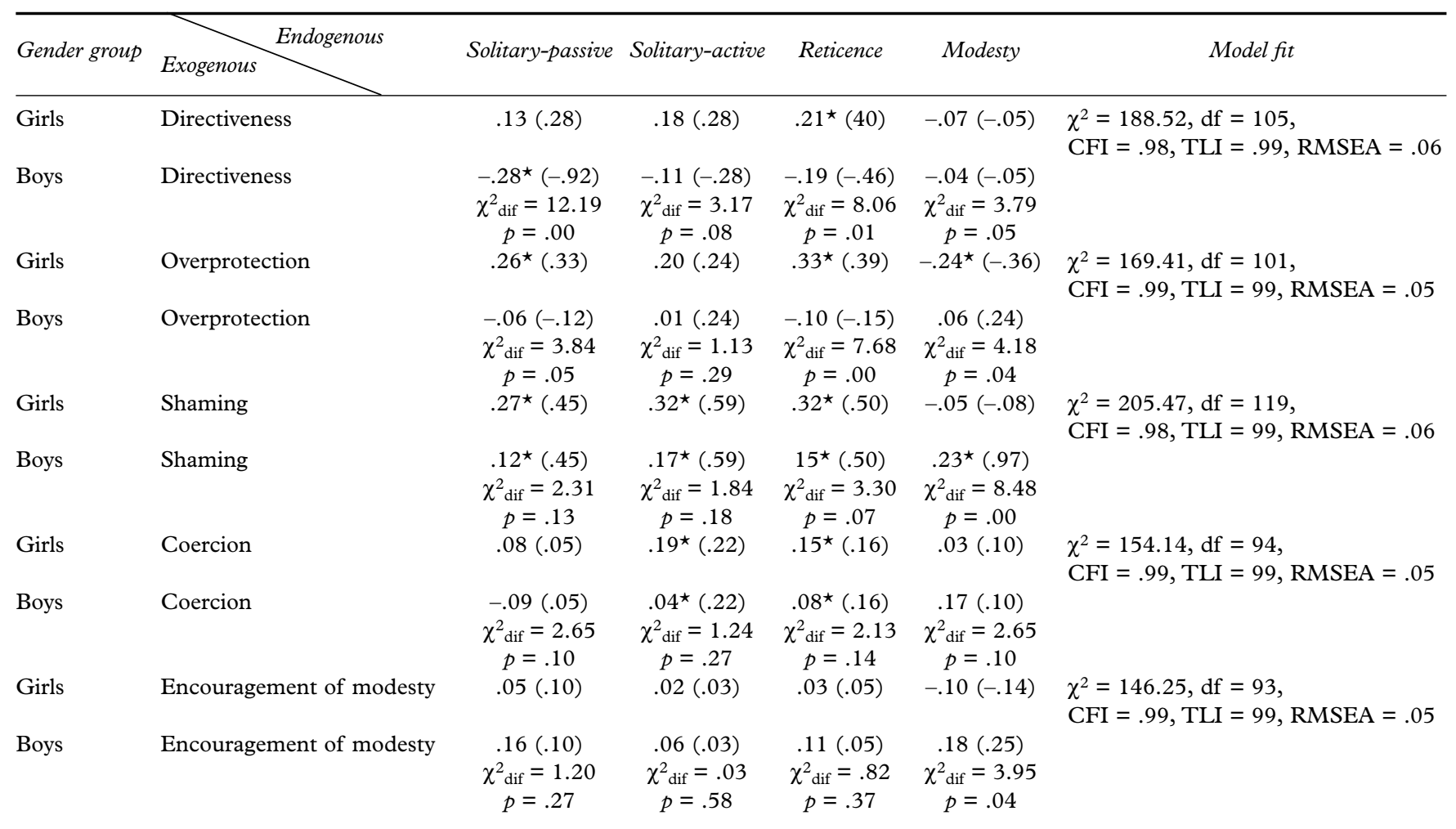

Note. The degree of freedom for each chi-square difference test is one. The chi-square difference is between a model with equality constraints on the path across the child gender group and another model without such equality constraints. Significance tests are based on the unstandardized coefficients (in parentheses) and their associated standard errors. ${ }^{\star} p<.05$. 
study were still found to be associated with child behaviors even after controlling for child age. Specifically, the path coefficients for the relations between the parenting practices and each respective child behavior (solitary-passive, reticent, solitary-active, and modest behaviors) and model fit indices are presented in Table 4.

The results suggested that directiveness was positively associated with girls' reticent behavior $(\gamma=.21, z=2.81, p<$ $.05)$; that overprotection was positively related to girls' solitary passive $(\gamma=.26, z=2.39, p<.05)$, reticence $(\gamma=.33, z=2.81$, $p<.05)$ and negatively related to modesty $(\gamma=-.24, z=2.42$, $p<.05)$ for girls; that shaming was positively associated with solitary-passive behavior $(\gamma=.27, z=3.29, p<.05)$, solitaryactive behavior $(\gamma=.32, z=3.84, p<.05)$, and reticence $(\gamma=$ $.32, z=4.01, p<.05)$ for girls, and with solitary-passive behavior $(\gamma=.12, z=3.29, p<.05)$, solitary-active behavior $(\gamma=.17, z=3.84, p<.05)$, reticence $(\gamma=.15, z=4.01, p<$ $.05)$ and modest behavior $(\gamma=.23, z=4.00, p<.05)$ for boys; and that coercion was positively associated with solitary-active behavior $(\gamma=.19, z=2.29, p<.05)$ and reticence $(\gamma=.15$, $z=2.18, p<.05)$ for girls. In addition, child age was negatively associated with girls' reticence $(\gamma=-.19, z>2.00, p<$ $.05)$ and positively associated with boys' modesty $(\gamma=.20, z>$ $2.00, p<.05)$ in overall. No relations were found for encouragement of modest behavior.

Results also showed that several paths were not equivalent across groups (see Table 4). Specifically, in regard to directiveness, the effect for boys was significantly more negative for solitary-passive $\left(\chi_{\text {dif }}^{2}=12.19, p<.01\right)$ and reticent $\left(\chi_{\text {dif }}^{2}=\right.$ $8.06, p<.05)$ behaviors than it was for girls. In regard to overprotection, compared to boys, the effect for girls was significantly more positive for reticence $\left(\chi_{\text {dif }}^{2}=7.68, p<.01\right)$, and significantly more negative for modesty $\left(\chi_{\text {dif }}^{2}=4.18, p<.05\right)$. In regard to shaming, the effect for boys was significantly more positive for modesty $\left(\chi_{\text {dif }}^{2}=8.48, p<.01\right)$ than it was for girls. Finally, in regard to encouragement of modesty, the effect for boys was significantly more positive for modesty $\left(\chi_{\text {dif }}^{2}=3.95\right.$, $p<.05)$ than it was for girls.

\section{Discussion}

The goal of this study was to examine the association between specific parenting practices (i.e., encouragement of modest behavior, overprotection, shaming, directiveness, and coercion), and child behavior including subtypes of social withdrawal (i.e., reticence, solitary-passive withdrawal, solitary-active behavior), and modest behavior in early childhood in China. Results showed that (a) maternal directiveness was positively associated with reticent behavior in girls and negatively associated with solitary-passive behavior in boys, (b) maternal overprotection, for girls, was positively related to both reticent behavior and solitary-passive behavior, and negatively related to modest behavior (c) coercion was positively associated with solitary-active and reticent behavior in girls, and (d) shaming was positively related to all forms of withdrawn behaviors in boys and girls, as well as positively related to modest behavior in boys. These findings identify links between parenting practices and social withdrawal in China that are consistent with findings in western cultures such as the United States, as well as unique to China. Because the correlational nature of this study precludes conclusions regarding causality, these findings may underscore both the role that parents may play in the development of socially withdrawn behaviors in China as well as the potential influence that child behaviors may have on parenting. Hence, the meaningful contributions of this study to our current understanding of the bi-directional nature of parenting practices and child social withdrawal in early childhood in China will be discussed in turn.

The first notable finding pertains to the significant association between all forms of intrusive parenting practices (directiveness, overprotection, and coercion) and reticent behavior for girls. To an extent, this mirrors what researchers have found in Western cultures. Specifically, researchers have found that mothers who give unsolicited direction to their children on how they should act (i.e., directiveness), or who are overprotective, or who are harsh in their parenting (Baumrind, 1971), tend to have shy, reticent children at age 4 (e.g., Coplan et al., 2004; Rubin et al., 1999, 2002). Given that this was the first attempt to examine the association between these parenting practices and withdrawn subtypes in early childhood in China, the findings advance our current knowledge by demonstrating that the link between intrusive parenting and reticent behavior appears to cross cultural boundaries.

As previously noted, the correlational design employed in this study precludes causal inferences. Thus, it is not possible to assess whether parents are reacting to their children's shyness or if parental behavior is causing fearfulness. On the one hand, intrusive parenting could lead to withdrawn behavior. As noted previously, Rubin et al. (2002) found that if mothers of inhibited toddlers (age 2) engaged in intrusive parenting behaviors (e.g., unsolicited intervention) they tended to have children who engaged in higher amounts of reticent/wary behavior at age 4 than did the children who, as inhibited toddlers, did not have mothers who engaged in oversolicitous parenting.

On the other hand, there is considerable reason to believe that children's display of reticent behavior evokes overcontrolling behaviors from parents. Research suggests that children who display reticent behavior may have an underlying dispositional or biological "make-up" (e.g., Fox et al., 1995; Reznick et al., 1985; Tout, de Haan, Campbell, \& Gunnar, 1998). Thus, it may be that parents are responding to wariness in their children. Indeed, Rubin et al. (1999) found that perceived wariness at age 2 predicted overprotective parenting at age 4 while the path between overprotective parenting at age 2 and shy behaviors at age 4 was not significant.

Taken together, mothers may be responding to perceived wariness in their children with parental behaviors (i.e., intrusive, overprotective parenting) aimed at helping their children interact socially. Unfortunately, rather than helping their children, mothers may actually exacerbate the problem by not allowing children opportunities to develop regulatory and coping skills to deal with their social anxieties. However, that may not be a goal of Chinese mothers. In other words, helping a child's own psychological state may not be the primary focus of Chinese mothers. Cheah and Rubin (2004) gave mothers scenarios in which their children displayed wary behavior and asked them to describe how that behavior made them feel, how they would respond, and why. Mothers reported that they would respond to withdrawal with anxiety, puzzlement, disappointment, and anger. Compared to European American mothers, Chinese mothers chose more directive strategies aimed at helping their children know how to behave in the future and how to fit in with the group. In other words, as 
noted previously, Chinese mothers appeared to be more concerned with how a child acted than how he or she felt. Results of the present study support that notion in that parenting practices aimed at correcting behavior (e.g., "tell our child how he/she should behave", "tell child that he/she should be ashamed when misbehaving", "slap our child when the child misbehaves") were consistently linked to reticence.

Again, however, it is important to reiterate the caveat that the relations between reticence and intrusive parenting were found only for girls. At first glance, these findings appear to contradict what has been found in western cultures. For example, in recent years, there has been a growing body of evidence in Western cultures suggesting that shyness is more likely to be accepted in girls than in boys (see Burgess et al., 2001). Results from the present study may actually support this notion. While there was a relation between the use of directiveness, overprotection, coercion, and shaming, respectively, and girls' reticent behavior, only shaming was related to boys' reticent behavior. It may be that mothers are more willing to simply guide and direct a daughter's behavior but will intervene more harshly with shaming when it comes to the same behavior in boys. This may reflect a greater concern in regard to reticence in boys than girls. However, this explanation is only speculative and, again, cannot be confirmed via the correlational nature of this study. Furthermore, the magnitude of the relations between reticence and all intrusive parenting practices as well as shaming in girls cannot be overlooked. Future work needs to build on these findings by exploring how parents feel about reticence in boys compared to girls and the socialization goals they have for each.

Another notable finding pertains to the relations between parenting practices and solitary-active withdrawal. Results showed a relation between solitary-active behavior and both shaming and coercion. Solitary-active behavior tends to reflect immaturity and impulsivity (e.g., Rubin, 1982) rather than either social wariness or disinterest. Children who exhibit this behavior tend to be isolated from and by the peer group. These findings again lead to questions of causality. In regard to reticence, we speculated that parents may be responding to their children's wary predispositions. In the case of solitary-active behavior, there is evidence that would suggest that the direction of effects goes the other way. There is an extensive body of literature linking harsh, coercive parenting to child externalizing disorders (see Hart et al., 2003, for a review). Thus, parents who rely on spanking, grabbing, and slapping to socialize children may not equip them with the skills necessary to interact socially. As a result, their children would engage in the type of immature and impulsive behaviors that lead to exclusion by peers. While future work is needed to explore the direction of effects, the results of the present study underscore the link between negative parenting practices and solitaryactive withdrawal in China.

The next set of notable findings focus on the association between maternal shaming and child behaviors. Specifically, results revealed a positive relation between shaming and every form of social withdrawal for boys and girls as well as a positive association with modesty in boys. It is believed that shaming is widely used by Chinese parents to motivate children to take responsibility for their actions (Fung, 1999). In particular, parents may be concerned about behavior that detracts from participation in and contributing to group processes (Stimpfl, Zheng, \& Meredith, 1997). Indeed, Chinese parents appear to perceive both immodesty (e.g., Fung, 1999) and social with- drawal (e.g., Cheah \& Rubin, 2004), in particular reticence, to be problematic. Furthermore, Chinese parents may care less about the reasons for withdrawal (i.e., fear, anxiety) and more simply about achieving the desired behavior (i.e., social participation). Thus, they may use shaming to change their child's behavior. In other words, children's withdrawal and immodesty may evoke parents' use of shaming. This would seem more logical than parents shaming their children into behaviors (i.e., reticence and immodesty) that they do not value (e.g., Cheah \& Rubin, 2004).

Arguments for possible causal directions for the relation between shaming and solitary-passive behavior are more difficult to make. It may very well be that solitary-passive behavior elicits parental use of shame because it is perceived to be harmful to group harmony because the child is engaged in nonsocial behavior. However, solitary-passive behavior does not appear to harm peer relationships in early childhood in China (Hart et al., 2000) and, therefore, parents may not view it negatively. In fact, parents may actually value this behavior and, therefore, try to foster it. Specifically, because Chinese parents value academic achievement (e.g., Chao, 2001; Ho, 1986), solitary-passive withdrawal may be perceived as a behavior that is conducive to academic performance because it focuses on quiet, constructive and explorative behaviors which are preferred behaviors in the classroom. Furthermore, research on solitary-passive withdrawal in western cultures suggests that children who demonstrate this behavior tend to exhibit a degree of social competence in peer settings (e.g., Coplan et al., 1994; Coplan \& Rubin, 1998; Rubin, 1982; Rubin et al., 1995). Thus, parents might value solitary-passive behavior because it may foster appropriate learning behavior without compromising group relationships. Thus, solitarypassive behavior may be the product of parental shaming aimed at socializing behavior conducive to academic achievement.

Finally, these findings also underscore the need to keep the constructs of withdrawal distinct from the construct of modesty. For several years, there have been apparent discrepancies regarding whether or not withdrawal is valued in China. For example, Chen and colleagues found shyness to be linked to positive outcomes such as social maturity and competence, leadership, peer acceptance, and self-mastery (Chen et al., 1992, 1995) while others (e.g., Hart et al., 2000; Schwartz, et al., 2001) found withdrawal to be negatively related to peer acceptance. While these findings may be linked to differences in age (some of Chen's work examined adolescents, Schwartz and colleagues studied children, Hart and colleagues studied preschoolers), it is more likely that the different findings are related to different constructs. As noted in the introduction, some constructs appear to reflect oversensitivity (e.g., Chen et al., 1992), while others reflect social wariness (Hart et al., 2000). There is significant research documenting the value placed on social interaction and relationships in China (see Oyserman, Coon, \& Kemmelmeier, 2002, for a review) and, therefore, researchers need to clearly differentiate between behaviors that foster social relationships (e.g., modest behaviors) and those that do not (e.g., wariness). Withdrawing from the peer group due to fear would be perceived as harmful in a culture that values the group, while being humble, submissive, academically oriented, and sensitive to how others perceive you would foster relationships in a group. Therefore, effort needs to be made to avoid blending competing constructs. The results of this study (e.g., a positive relation between overprotection and reticence, and a negative relation between 
overprotection and modesty) further show that modesty and reticence appear to be distinct constructs with different parenting correlates.

Taken together, these studies suggest that some parents may respond to perceived wariness in their children with parental behaviors (i.e., intrusive, overprotective parenting) that actually reinforce the problem by not allowing children opportunities to develop regulatory and coping skills to deal with their social anxieties. Furthermore, parents may use shaming to promote behaviors they value and change behaviors they do not. It is unfortunate that the correlational nature of this study precludes the ability to shed greater light on which of these potential causal explanations may be more accurate. The results of this study are certainly only preliminary in nature. They are, however, an essential first step in identifying linkages between certain parenting practices and forms of social withdrawal in China. These findings demonstrate the importance of examining the bi-directional influence of parenting and child behaviors, and provide a foundation from which future work examining these associations can build.

\section{Limitations and future directions}

While this study provides a better understanding of the relations between parenting practices and social withdrawal in China, there are several limitations as well. First, the study focused only on mothers. Fathers may exhibit different parenting practices than mothers with different influences on child behaviors. For example, Yang et al. (2004) found that fathers tend to be more coercive and psychologically controlling with daughters than are mothers, and these differences in parenting are related to various forms of aggression. More work is needed in order to understand the role that fathers may play in the development of social withdrawal. Second, parenting practices were based only on mothers' self-reports. There is an old Chinese saying: "The bad thing that happened at home should not be told to others." Any time self-report data are used there is the possibility that individuals answer the way they believe they should answer rather than truthfully, but that is of particular concern in a culture in which there is concern about bringing shame to the group (i.e., family). Thus, future work might include observations or, at least, reports from spouses. Third, the magnitude of the relations in this study were modest, at best. Thus, interpretations should be made cautiously and with the understanding that the parent-child relationship is just one of several factors (e.g., temperament, self-perceptions, etc.) influencing withdrawal in early childhood. Next, the use of teacher ratings typically results in larger correlations between the subtypes of withdrawal than does the use of observational methodologies. Given that few observational studies of young children's withdrawn behaviors have been conducted in China, caution is warranted in comparing findings using teacher measures in China with those of observational studies conducted in western cultures. Future work should include observational approaches to studying nonsocial behaviors in China. Finally, participants in this sample resided in Beijing and Hangzhou and their responses may not be representative of mothers in other geographic areas in mainland China.

Despite these limitations, this study advances our understanding of the relations between parenting practices and preschoolers' social withdrawal in China. The findings highlight similarities to research done in Western cultures (e.g., overprotective parenting is associated with reticence) as well as results which appear to be unique to China (e.g., shaming is related to solitary-passive behavior).

\section{References}

Asendorpf, J. (1990). Beyond social withdrawal: Shyness, unsociability and peer avoidance. Child Development, 33, 250-259.

Barber, B.K. (2002). Intrusive parenting: How psychological control affects children and adolescents. Washington, DC: American Psychological Association.

Baumrind, D. (1967). Child care practices anteceding three patterns of preschool behavior. Genetic Psychology Monographs, 75, 43-88.

Baumrind, D. (1971). Current patterns of parental authority. Developmental Psychology Monographs, 4, 1-103.

Berry, J.W. (1989). Imposed etics-emics-derived etics: The operationalisation of a compelling idea. International fournal of Psychology, 24, 721-735.

Bollen, K.A. (1989). Structural equations with latent variables. New York: John Wiley.

Burgess, K.B., Rubin, K.H., Cheah, C.S.L., \& Nelson, J.L. (2001). Behavioral inhibition, social withdrawal, and parenting. In W.R. Crozier \& L.E. Alden (Eds), International Handbook of Social Anxiety: Concepts, research, and interventions relating to the self and shyness (pp. 137-158). Chichester: Wiley.

Chao, R.K. (1994). Beyond parental control and authoritarian parenting style: Understanding Chinese parenting through the cultural notion of training. Child Development, 65, 1111-1119.

Chao, R.K., \& Sue, S. (1996). Chinese parental influence and their children's school success: A paradox in the literature on parenting styles. In S. Lau (Ed.), Growing up the Chinese way (pp. 93-120). Hong Kong: The Chinese University Press.

Chao, R.K. (2001). Extending research on the consequences of parenting style for Chinese Americans and European Americans. Child Development, 72, 1832-1843.

Chao, R.K., \& Tseng, V. (2002). Parenting of Asians. In M.H. Bornstein (Ed.), Handbook of parenting. Volume 4: Social conditions and applied parenting (pp. 59-93). Mahwah, NJ: Lawrence Erlbaum.

Chang, L., Lansford, J.E., Schwartz, D., \& Farver, J.M. (2004). Marital quality, maternal depressed affect, harsh parenting, and child externalizing in Hong Kong Chinese families. International fournal of Behavioral Development, 28, 311-318.

Cheah, C.S.L., \& Rubin, K.H. (2004). European and mainland Chinese mothers' responses to aggression and social withdrawal in preschoolers. International fournal of Behavioral Development, 28, 83-94.

Chen, X., Cen, G., Li, D., \& He, Y. (2005). Social functioning and adjustment in Chinese children: The imprint of historical time. Child Development, 76, $182-195$.

Chen, X., Dong, Q., \& Zou, H. (1997). Authoritative and authoritarian parenting practices and social school performance in Chinese children. International fournal of Behavioral Development, 21, 835-873.

Chen, X., Liu, M., \& Li, D. (2000). Parental warmth, control, and indulgence and their relations to adjustment in Chinese children: A longitudinal study. fournal of Family Psychology, 14, 401-419.

Chen, X., Liu, M., Li, D., Cen, G., Chen, H., \& Wang, L. (2000). Maternal authoritative and authoritarian attitudes and mother-child interactions and relationships in urban China. International fournal of Behavioral Development, 24, 119-126.

Chen, X., Rubin, K.H., Cen, G., Hastings, P.D., Chen, H., \& Stewart, S.L. (1998). Child-rearing attitudes and behavioral inhibition in Chinese and Canadian toddlers: A cross-cultural study. Developmental Psychology, 34, 677-686.

Chen, X., Rubin, K.H., \& Li, Z. (1995). Social functioning and adjustment in Chinese children: A longitudinal study. Developmental Psychology, 31, 531-539.

Chen, X., Rubin, K.H., \& Sun, Y. (1992). Social reputation and peer relationships in Chinese and Canadian children: A cross-cultural study. Child Development, 63, 1336-1343.

Chen, X., Wu, H., Chen, H., Wang, L., \& Cen, G. (2001). Parenting practices and aggressive behavior in Chinese children. Parenting: Science and Practice, 1, 159-184.

Coenders, G., Satorra, A., \& Saris, W.E. (1997). Alternative approaches to structural modeling of ordinal data: A Monte Carlo study. Structural Equation Modeling, 4, 261-282.

Coplan, R.J., \& Rubin, K.H. (1998). Exploring and assessing non-social play in the preschool: The development and validation of the Preschool Play Behavior Scale. Social Development, 7, 72-91. 
Coplan, R.J., Molina, M.G., Lagace-Seguin, D.G., \& Wichmann, C. (2001).When girls versus boys play alone: Gender differences in the relations between nonsocial play and adjustment in kindergarten. Developmental Psychology, 37, 464-831.

Coplan, R.J., Prakash, K., O’Neil, K., \& Armer, M. (2004). Do you “want” to play? Distinguishing between conflicted shyness and social disinterest in early childhood. Developmental Psychology, 40, 244-258.

Coplan, K.J., Rubin, K.H., Fox, N.A., Calkins, S.D., \& Stewart, S.L.(1994). Being alone, playing alone, and acting alone: Distinguishing among reticence and passive and active solitude in young children. Child Development, 65, 129-137.

Darling, N., \& Steinberg, L. (1993). Parenting style as context: An integrative model. Psychological Bulletin, 113, 487-496.

Fox, N.A, Rubin, K.H., Calkins, S.D., Marshall, T.R., Coplan, R.J., Porges, S.W., Long, J.M., \& Stewart, S. (1995). Frontal activation asymmetry and social competence at four years of age. Child Development, 66, 1770-1784.

Fung, H. (1999). Becoming a moral child: The socialization of shame among young Chinese children. Ethos, 27, 180-209.

Hart, C.H., DeWolf, M.D., \& Burts, D.C. (1993). Parental discipline strategies and preschoolers' play behavior in playground settings. In C.H. Hart (Ed.), Children on playgrounds: Research perspectives and applications (pp. 271-313). Albany: State University of New York Press.

Hart, C.H., Newell, L.D., \& Olsen, S.F. (2003). Parenting skills and social/communicative competence in childhood. In J.O. Greene \& B.R. Burleson (Eds.). Handbook of Communication and Social Interaction Skill. Mahwah, NJ: Erlbaum.

Hart, C.H., Yang, C., Nelson, D.A., Jin, S., Bazarskaya, N., Nelson, L.J., et al. (1998). Peer contact patterns, parenting practices, and preschoolers' social competence in China, Russia, and the United States. In P. Slee \& K. Rigby (Eds.), Children's peer relations (pp. 3-30). London: Routledge.

Hart, C.H., Yang, C., Nelson, L.J., Robinson, C.C., Olsen, J.A., Nelson, D.A., Porter, C.L., Jin, S., Olsen, S.F., \& Wu, P. (2000). Peer acceptance in early childhood and subtypes of socially withdrawn behavior in China, Russia and the United States. International fournal of Behavioral Development, 24, 73-81.

Ho, D.Y.F. (1986). Chinese pattern of socialization: A critical review. In M.H. Bond (Ed.), The psychology of the Chinese people (pp. 1-37). Oxford: Oxford University Press.

Ho, M.K. (1987). Family therapy with minorities. Newbury Park, CA: Sage.

Kline, R.B. (1998). Principles and practice of structural equation modeling. New York: The Guilford Press.

Komaroff, E. (1997). Effect of simultaneous violations of essential tau equivalence and uncorrelated errors on coefficient alpha. Applied Psychological Measurement, 21, 337-348.

Lau, S. (1996). Growing up in the Chinese way: Chinese child and adolescent development. Hong Kong: The Chinese University Press.

Leung, K., Lau, S., \& Lam, W. (1998). Parenting styles and academic achievement: A cross-cultural study. Merrill-Palmer Quarterly, 44, 157-172.

Lin, C.C, \& Fu, V.R. (1990). A comparison of child-rearing practices among Chinese, immigrant Chinese, and Caucasian-American parents. Child Development, 61, 429-433.

Lubke, G.H., \& Muthén, B.O. (2004). Applying multigroup confirmatory factor models for continuous outcomes to Likert scale data complicates meaningful group comparisons. Structural Equation Modeling, 11(4), 514-534.

Muthén, L.K., \& Muthén, B.O. (2001). Mplus user's guide (3rd ed.), Los Angeles, CA: Muthen \& Muthen.

Nelson, D.A., Hart, C.H., Yang, C., Olsen, J.A., \& Jin, S. (2006). Aversive parenting in China: Associations with child physical; and relational aggression. Child Development, 77, 554-572.

Nelson, D.A., Nelson, L.J., Hart, C.H., Yang, C., \& Jin, S. (2006). Parenting and peer group behavior in cultural context. In X. Chen, D. French, \& B. Schneider (Eds.), Peer relations in cultural context (pp. 213-246). New York: Cambridge University Press.

Oyserman, D., Coon, H.M., \& Kemmelmeier, M. (2002). Rethinking individualism and collectivism: Evaluation of theoretical assumptions and metaanalyses. Psychological Bulletin, 128, 3-72.

Porter, C.L., Hart, C.H., Yang, C., Zeng, Q., Robinson, C.C., Olsen, J.A., Olsen, S.F., \& Jin, S. (2005). A comparative study of child temperament and parenting in Beijing, China, and the Western United States. International fournal of Behavioral Development, 29, 541-551.
Reznick, J.S., Kagan, J., Snidman, N., Gersten, M., Baak, K., \& Rosenberg, A. (1985). Inhibited and uninhibited children: A follow-up study. Child Development, 57, 660-680.

Rigdon, E.E., \& Ferguson, C.E. Jr. (1991). The performance of the polychoric correlation coefficient and selected fitting functions in confirmatory factor analysis with ordinal data. fournal of Marketing Research, 28, 491-497.

Rubin, K.H. (1982). Nonsocial play in preschoolers: Necessarily evil? Child Development, 53, 651-657.

Rubin, K.H., \& Mills, R.S.L. (1988). The many faces of social isolation in childhood. Fournal of Consulting and Clinical Psychology, 56, 916-924.

Rubin, K.H., Burgess, K.B., \& Coplan, R.J. (2002). Social withdrawal and shyness. In P.K. Smith \& C.H. Hart (Eds.), Blackwell handbook of child social development (pp. 329-352). Cornwall: Blackwell.

Rubin, K.H., Burgess, K.B., \& Hastings, P.D. (2002). Stability and socialbehavioral consequences of toddlers' inhibited temperament and parenting behaviors. Child Development, 73, 483-495.

Rubin, K.H., Bukowski, W., \& Parker, J. (1998). Peer interactions, relationships, and groups. In N. Eisenberg (Ed.), Handbook of child psychology: Social, emotional, and personality development (5th ed., pp. 619-700). New York: Macmillan.

Rubin, K.H., Coplan, R.J., Fox, N.A., \& Calkins, S.D. (1995). Emotionality, emotion regulation, and preschoolers' social adaptation. Development and Psychopathology, 7, 49-62.

Rubin, K.H., Hymel, S., \& Mills, R.S. (1989). Sociability and social withdrawal in childhood: Stability and outcomes. Fournal of Personality, 57, 237-255.

Rubin, K.H., Nelson, L.J., Hastings, P., \& Asendorpf, J. (1999). The transaction between parents' perceptions of their children's shyness and their parenting styles. International fournal of Behavioral Development, 23, 937-958.

Schmitt, N. (1996). Uses and abuses of coefficient alpha. Psychological Assessment, 8, 81-84.

Schoenhals, M. (1993). The paradox of power in a People's Republic of China middle school. Armonk, NY: M.E. Sharpe.

Schwartz, D., Chang, L., \& Farver, J.M. (2001). Correlates of victimization in Chinese children's peer groups. Developmental Psychology, 37, 520-532.

Simpson, A.E., \& Stevenson-Hinde, J. (1985). Temperamental characteristics of three-to-four-year-old boys and girls and child-family interactions. Fournal of Child Psychology and Psychiatry, 26, 43-53.

Spinrad, T.L., Eisenberg, N., Harris, E., Hanish, L., Fabes, R.A., Kupanoff, K., Ringwald, S., \& Holmes, J. (2004). The relation of children's everyday nonsocial peer play behavior to their emotionality, regulation, and social functioning. Developmental Psychology, 40, 67-80.

Steinberg, L., Lamborn, S.D., Darling, N., Mounts, N.S., \& Dornbusch, S.M. (1994). Over-time changes in adjustment and competence among adolescents from authoritative, authoritarian, indulgent, and neglectful families. Child Development, 65, 754-770.

Stevens, J. (1996). Applied multivariate statistics for the social sciences. Mahwah, NJ: Lawrence Erlbaum.

Stimpfl, J., Zheng, F., \& Meredith, W. (1997). A garden in the motherland: A study of a preschool in China. Early Child Development and Care, 129, 11-26.

Tout, K., De Haan, M., Campbell, E.K., \& Gunnar, M.R. (1998). Social behavior correlates of cortisol activity in child care: Gender differences and time-of-day effects. Child Development, 69, 1247-1262.

Tseng, W.W., \& Wu, D.Y.H. (1985). Introduction: The characteristics of Chinese culture. In W.S. Tseng and D.Y.H. Wu (Eds.), Chinese cultural and mental health (pp. 3-13). Orlando, FL: Academic Press.

Wright, B.D. (1999). Fundamental measurement of psychology. In S.E. Embreston \& S.L. Hershberger (Eds.), The new rules of measurement: What every psychologist and educator should know (pp. 65-104). Mahwah, NJ: Lawrence Erlbaum.

Wu, P., Robinson, C.C., Yang, C., Hart, C.H., Olsen, S.F., Porter, C.L., Jin, S., Wo, J., \& Wu, X. (2002). Similarities and differences in mother's parenting of preschoolers in China and the United States. International fournal of Behavioral Development, 26, 481-491.

Yang, C., Hart, C.H., Nelson, D.A., Porter, C.L., Olsen, S.F., Robinson, C.C., \& Jin, S. (2004). Fathering in a Beijing, Chinese sample: Associations with boys' and girls' negative emotionality and aggression. In R.D. Day \& M.E. Lamb (Eds.), Conceptualizing and measuring father involvement (pp. 185-216). Mahwah, NJ: Erlbaum. 